\title{
Social Distance Modeling on the Copenhagen, Denmark, Metro
}

\author{
Bilde, Basthiann A.; Andersen, Morten L.; Harrod, Steven
}

Published in:

Journal of Transportation Engineering Part A: Systems

Link to article, DOI:

10.1061/JTEPBS.0000633

Publication date:

2022

Document Version

Peer reviewed version

Link back to DTU Orbit

Citation (APA):

Bilde, B. A., Andersen, M. L., \& Harrod, S. (2022). Social Distance Modeling on the Copenhagen, Denmark, Metro. Journal of Transportation Engineering Part A: Systems, 148(2), [e0000633].

https://doi.org/10.1061/JTEPBS.0000633

\section{General rights}

Copyright and moral rights for the publications made accessible in the public portal are retained by the authors and/or other copyright owners and it is a condition of accessing publications that users recognise and abide by the legal requirements associated with these rights.

- Users may download and print one copy of any publication from the public portal for the purpose of private study or research.

- You may not further distribute the material or use it for any profit-making activity or commercial gain

- You may freely distribute the URL identifying the publication in the public portal

If you believe that this document breaches copyright please contact us providing details, and we will remove access to the work immediately and investigate your claim. 


\title{
Social Distance Modelling on the Copenhagen Metro
}

\author{
Basthiann A. Bilde a , Morten L. Andersen ${ }^{\text {b }}$, Steven Harrod ${ }^{1, \mathrm{c}}$ \\ ${ }^{a}$ Consultant, Atkins Denmark, Arne Jacobsens Allé 17, 2300 København, Denmark: \\ Basthiann.Bilde@atkinsglobal.com \\ bIndependent, Bjergegade 30b 1. tv 3000 Helsingør, Denmark: morten.1.a@outlook.dk \\ 'Associate Professor, Technical University of Denmark, Lautrupvang 15, 2750 Ballerup, Denmark \\ ${ }^{1}$ Corresponding author: stehar@dtu.dk, Phone: +45 51862861
}

\begin{abstract}
Public transport is a critical service in Copenhagen, because many residents do not own a car, and in any event, car travel is not practical in the city center due to narrow roads and lack of parking. In response to COVID-19, Danish public health authorities have established a minimum 1 meter social distancing policy in public spaces. This study simulates passenger pedestrian flow in three representative stations of the Copenhagen metro to determine if these goals can be attained, and if any physical changes should be made. The study is conducted with a micro-simulation in Bentley Legion of the passenger flow in three representative stations, with small, medium, and large traffic flows. The simulation is agent-based, and the individual objective function is minimum cost according to walking distance, comfort, and frustration.

The results show that for the majority of stations, the physical infrastructure and the expected traffic flow are compatible with the social distancing goals. However, for a few of the highest demand stations, particularly those that serve as intermodal hubs, there are great difficulties in achieving the desired social distancing measures. In particular, the intermodal hub station of Nørreport does not possess corridors and escalators which are distributed correctly according to the pedestrian flow. This station is underground and it is unfortunately not easy to change this infrastructure.
\end{abstract}

\section{Keywords}

COVID, Pedestrian Simulation, Public Transit Stations

\section{Introduction}

Over the past year, the world has felt the effects of the COVID-19 pandemic, and the public transport systems have been hit especially hard. In Copenhagen, public transport has experienced a large decline in demand followed by a gradual increase to a stable demand of about half the level prior to COVID-19. At the same time, public health authorities have established requirements for social distancing, or greater open space around each passenger. The research question in this paper is whether these social distancing goals can be reasonably met within the existing infrastructure or with minor alterations.

This paper addresses how these parameters and restrictions have effected the M1/M2 metro lines of Copenhagen, as well as shed some light on what can be done to improve the passenger flow within stations under COVID-19 guidelines. The responsible operating authority, Metroselskabet I/S, has provided actual data on passenger flows and operations for a half year period stretching from before the COVID-19 outbreak to the recover from the first infection wave. Metroselskabet controls multiple passenger-counting sensors installed at all the 
stations, which provides for very detailed physical body counts within the stations. From this data, three representative stations have been selected for deeper analysis.

The physical flows of passengers within the stations have been simulated using the commercial software Bentley Legion. The simulations with Bentley Legion provide a complete analysis of each station. The output clearly identifies problematic zones and bottlenecks by mapping results such as passenger density, social distance breaks and overall space utilization. Additionally, the simulation models offer data to determine doorway utilization. The results possibly offer a theoretical approach to determine potential dwell time reductions, which naturally offer possibilities to achieve savings in rolling stock circulation or offer other possibilities to re-distribute rolling stock.

Several international studies have been consulted to determine specific parameters such as average passenger space requirements and walking speed distributions. Three stations have been selected from the M1/M2 routes to represent three levels of station size, where station size is measured by passenger counts. These representative stations are Nørreport (large station), Forum (medium), and Øresund (small).

The results generally show that COVID-19 does not create any significant passenger flow issues for Forum Station and Øresund Station, but a string of issues do arise in Nørreport Station. In addition, the results point to a general improvement of the passenger flow, going from before COVID-19 to during COVID-19. Though when looking into a COVID-19 scenario with ridership returning to a higher level, many of the same tendencies and complications appear, as seen before the COVID-19 outbreak, but with increased effect. It will be difficult to make day-to-day, small investment, improvements to the stations, because the analysis shows a great need to expand platform space and increase capacity in escalators. However, design choices like these can be considered in future station designs, to accommodate for future pandemics by making the infrastructure more robust.

\section{Literature review}

A variety of literature concerning pedestrian flow in stations exists, mostly concerned with capacity or passenger comfort. Azadpeyma and Kashi (2019) apply VISSIM software to a pedestrian study of Shohada Station in Iran. Pedestrian congestion is measured according to the Level of Service defined by Transportation Research Board (2013), and the analysis finds that removing ticket sales counters and increasing train frequency will reduce pedestrian congestion. Duives et al. (2013) provides a comprehensive survey of published pedestrian simulation models but specifically excludes commercial software.

Bentley Legion is an established pedestrian simulation tool that has until recently been the preferred pedestrian simulation module for Siemens AIMSUN traffic simulation software. Alexandersson and Johansson (2013) perform a comparative analysis of the performance of pedestrian similators Viswalk and Legion for AIMSUN (Bentley Legion), and find that the two products return similar results without any notable faults. Dubroca-Voisin et al. (2019) performs a review of nine pedestrian simulator tools, including Bentley Legion, and finds it difficult to distinguish between their capabilities for application in railway station modeling.

Grontmij Carl Bro (2009) apply Bentley Legion software to a pedestrian study of the suburban and long distance train platforms of Nørreport station in Copenhagen. The purpose of this study was to minimize disruption to pedestrian flow while construction work was performed during service hours. Seriani and Fernandez (2015) apply Bentley Legion to the simulation of boarding and alighting at a metro vehicle door. Sun et al. (2016) apply 

Bentley Legion to evaluate the capacity of curved corridors in metro stations, and note that Bentley Legion has been used in China for various large-scale studies, such as the Beijing Olympic Games.

\section{Passenger behaviour and model design}

The dominant traffic during rush hour is commuters. Different traveler types have different physical behaviors and speeds, and for the sake of clarity and consistency of model results, all traffic is presumed to be commuters and only peak travel periods are modeled. Key performance measures (KPI) for the study are chosen following Pedersen and Center for Trafik og Transport (2003). These consist of gross passenger flow, average passenger flow, and a measure of door throughput. The definition of these measures may be summarized as:

\section{Passenger exchange (gross flow)}

Defined as: "The count of passengers entering and exiting the train through a door"

$$
F_{i}=E_{i}+A_{i}
$$

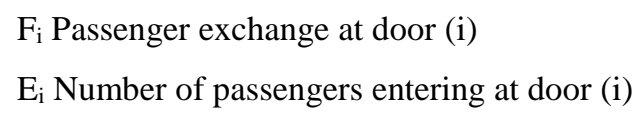

For the entire train at station (s) it is then

$$
F_{s}=\sum F_{i}
$$

\section{Average passenger exchange:}

The average passenger exchange time at a station for a single door:

$$
t_{t p}=\frac{t_{t}}{F_{i}}
$$

$t_{t p}$ Average passenger exchange time per passenger at a door (sec./pass.)

$t_{t} \quad$ Average door opening time (sec.)

$\mathrm{F}_{\mathrm{i}} \quad$ Passenger exchange at door (i)

\section{Door efficiency (throughput):}

Door efficiency measures the count of passengers entering/exiting the train per second. The door efficiency is the reciprocal of the average passenger exchange time.

$$
L_{i}=\frac{1}{t_{t p}}=\frac{F_{i}}{t_{t}}
$$

$\mathrm{L}_{\mathrm{i}} \quad$ Door efficiency for selected door (i) [Pass./sec.] 
Passengers vary greatly in their walking speed, and this of course affects their flow through the station, which then affects the quantity of passengers in the station (following from Little's Law (Little, 1961)), and finally determines the social distancing parameter of current concern. Herrstedt (2012) concludes from a literature survey that average pedestrian speed is 60 meters per minute, but the datasets contain large samples from elderly and youth populations. Finnis and Walton (2008) finds from a review of the literature and data collected in New Zealand that pedestrians adapt their speed to the environment, and that commuters have a higher expected speed.

Finnis and Walton observes activities between 8:15 a.m. and 5:15 p.m. on weekdays, at 4 separate locations, resulting in 1071 observations (Figure 1). These are unimpeded speed measurements. That is to say, the data only includes observations where the subject was not blocked or impeded. From this study, commuters have an average speed of 94.2 meter/minute with a $95 \%$ confidence interval (t-stat) of $(93.305,95.095)$. This is the fastest pedestrian category and statistically significant.

The Finnis and Walton study presents significantly faster values than the Herrstedt study. This favors results with lower passenger density in the station and potentially biases the results positively. However, the Finnis and Walton statistic is selected because it contains a more detailed data set and analysis. The simulation software, Bentley Legion, allows for individual passenger (entity) speeds to be drawn from a normal distribution. The values selected within the simulation model are a mean of 1.6 meters/second and a standard deviation of 0.2 , providing a cumulative distribution as shown in Figure 2, and corresponding to the Commuter values of Finnis and Walton.

Social distance is primarily measured as the radius distance between bodies. The desired distance standard from the Danish health authorities under COVID-19, during the study period, is 2 meters. Metroselkabet has anecdotally observed that this separation is nearly impossible to guarantee throughout the passenger's journey. This is especially evident onboard the trains since the rolling stock has a total width of 2.65 meters. On the platforms as well, social distancing was not part of the initial design parameters of the stations. Consequently, it is expected and experienced that passengers must accept a social distance less than 2 meters, because of the physical dimensions of the station.

Bentley Legion software implements passengers as a solid cylinder, so it is necessary to select a fixed dimension for passenger size. Jacobs (1967) provides a frequently cited reference for the volume of the most tightly packed crowd, where each person occupies $0.23 \mathrm{~m}^{2}$, or approximately a diameter of 0.55 meter. Sundhedststyrelsen (2020) recommends a minimum separation of 1 meter between persons in public spaces, and prefers 2 meters where possible and when there is known infection. The resulting model goal is then as shown in Figure 3.

Passengers movements are presumed to be "rational". That is, paths and choices of exit are presumed to follow a lowest cost model measured in time and effort. A comfort factor exists in the model that also encourages use of escalators and other conveniences. Passengers are also expected to wait in the marked standing areas at platform doors, which in the authors' personal experience is a regular pattern of behavior.

\subsection{Infrastructure}

The M1/M2 lines serve 22 stations, including three underground and six deep underground. Island platforms are standard throughout. The metro is an autonomous, driverless metro, and safety is ensured by the standard application of six platform doors for each track, which match the train doors in location and count. Station size is 
classified by traffic volume. Many of the stations share a common physical design, but there are physical differences at some stations and some unique features. This study has been conducted on three station classifications to get good representation if COVID-19's influence based on passenger numbers. The three classifications are as mentioned based on passenger numbers and are as following:

- Large station (more than 20,000 passengers/day)

- Medium station (between 7.000 and 20.000 passengers/day)

- Small station (less than 7.000 passengers/day)

The classification of the stations originates from Metroselskabet's traffic data from 2018 as seen in Figure 4. From the given limits, the distribution of the station classification is: 11 small stations, 9 medium stations, and 2 large stations.

Nørreport Station has been chosen to represent the large station class, because it is the absolute largest station based on Metroselskabet's own passenger numbers. Based on this, it is assumed that it is going to be most heavily influenced station. This is further backed by Nørreport Station's status as a major junction, between types of transport such as: bus, regional trains, S-trains, and the metro system. Nørreport Station will thereby mark the upper boundary for passenger load on the M1/M2 system. In addition to Nørreport Station, there is only one other station falling within the large station classification, Kongens Nytorv Station, which has approximately half the traffic of Nørreport Station.

Forum Station has been chosen to represent the medium station classification, since its physical design follows a common template very similar to Nørreport Station, except for a direct transfer tunnel, which connects to regional- and S-trains. The physical station design is additionally very similar to all the other deep underground stations on the M1/M2 lines, that primarily fall into the same size category as Forum Station.

Øresund Station has been chosen to represent the small station category, since it is an exemplary example of a small station on the M1/M2 network, serving a mostly residential neighborhood and without any significant connections to other transport modes. This is especially prominent when looking at the station design, with just one staircase and one elevator to service the passengers. It is relevant to note that Sundby Station is the station with the lowest passenger numbers on the network, but it has two staircases and one elevator, which makes it an outlier with higher capacity relative to the actual demand.

The rolling stock (trains) on lines M1/M2 are of a design from Hitachi Rail Italy Driverless Metro (previously AnsaldoBreda Driverless Metro). The trainsets consists of three coaches, two end coaches and one middle coach with extra space for luggage, cycles, and various strollers and wheelchairs. The trains are 39 meters long, consisting of 14 meters each in the end coaches and 11 meters in the middle coach, and have a top speed of 90 $\mathrm{km} / \mathrm{h}$. The trains are 2.65 meters wide and use a standard track gauge of 1,435 millimeters.

Every coach, regardless of being an end or middle wagon, has 2 doors on each side of the carriage, thus a complete train ( 3 cars) has 6 doors available for passenger exchange at a stop. Before COVID-19, the passenger capacity for each train was calculated as 4 passengers $/ \mathrm{m}^{2}$ + seating, but for the COVID-19 duration, Metroselskabet has set the capacity to $1-2$ passenger(s) $/ \mathrm{m}^{2}+$ seating. Considering that the total floor space is $58.7 \mathrm{~m}^{2}$ and there are 44 seats, the capacity before COVID-19 was 279 passengers/train, and 102-160 passengers/train during COVID-19, a reduction of $42.6 \%-73.4 \%$. This "capacity" measure is purely for planning purposes, as there are no reserved seats and no capacity controls at the stations. Metroselskabet's only possible action is to increase train frequency when traffic demand exceeds offered capacity. 


\subsection{Traffic demand and expected flows}

Metroselkabet conducted a private study in April 2019 over two weeks which revealed individual door utilization at the stations Nørreport, Kongens Nytorv, Christianshavn, Amagerbro, and Lergravsparken. This distribution of door preferences is asserted to be stable relative to traffic levels. This data is specific to each station, and reflects the physical design as well as the potential pedestrian destinations. The data was collected Monday to Thursday, during the morning rush between 7:20 and 8:20.

The stations Forum and Øresund are not included in this study. Their data is interpolated. Further counts were performed for Forum station by the authors to determine directional flow. Amagerbro station has the same design as Forum, so its door distribution data is used to represent the proportions between doors at Forum station.

Nørreport station door distribution data is used as a template for Øresund station. They are similar in that the majority of traffic at Nørreport chooses a single escalator and elevator. The directional distribution for Øresund is taken from the nearby Amagerbro station. The results for the three study stations are shown in Table 1.

The Copenhagen metro operates a frequency based service during most traffic hours, and does not follow a timetable. The peak (rush hour) period frequency is a departure interval of 104 seconds from Nørreport. Only half of these trains go to Øresund, so the interval there is 208 seconds. These values are applied in the simulation to determine passenger flows arriving and departing by train. Copenhagen metro has not modified train frequency during the reduction in demand due to COVID-19. Passenger demand scenarios consist of a pre-COVID-19 level based on data from 1-15 February 2020, a COVID-19 level based on 26-28 May 2020, and a hypothetical future scenario where traffic returns to $75 \%$ of pre-COVID-19 levels. The presumption in the future scenario is that COVID-19 is not resolved, but the long term restrictions on work and travel are not sustainable, and some compromise is required. Passenger demand is sampled from Tuesdays, Wednesdays, and Thursdays only. Denmark went into lockdown for the first time on March the $11^{\text {th }} 2020$. As shown in Figure 5, the passenger numbers started dwindling a little bit prior to the lockdown, and then after declined precipitously, dropping from more than 200,000 daily passengers to about 25,000. Ridership then slowly increases towards the summer of 2020, as a result of loosening restrictions and public acceptance of public transport after a mandatory facemask decree. From Figure 5, it can be seen that the 26-28 May period is representative of the traffic for the next few months. It is also appropriate because it is outside any holiday periods.

The distribution of traffic during the day is not clearly different during the COVID-19 period. Danish health authorities made strong recommendations to avoid rush hour travel and modify work hours, but the general ratio of rush hour to mid-day traffic is unchanged in the COVID-19 period. Figure 6 displays the hourly passenger counts for Nørreport station. Figure 7 presents a normalization of the hourly data, as a percentage of daily totals. There is a small reduction, and redistribution, of peak traffic, but the data sets do not have enough sample points to establish significance.

\section{Simulation and analysis in Bentley Legion}

Bentley Legion implements an agent-based simulation model with a minimum cost objective to calculate individual passenger (entity) routes. Readers who are unfamiliar with Bentley Legion may instead be familiar with AIMSUN simulator from Siemens. Until recently, the pedestrian simulator module in AIMSUN was in fact Bentley Legion. This analysis was performed in cooperation with the operator, Metroselskabet A/S, who also reviewed and validated the results. Bentley Legion has also been previously used at Nørreport station to study 
passenger flows to the suburban and long distance train platforms (Grontmij Carl Bro, 2009).

Passengers are presumed to make a rational decision according to time and some comfort factors (e.g. escalator vs. stairs). The objective function in Bentley Legion considers the following specific factors (Bentley, 2020):

- Frustration: being hindered in movement, slowed down, for example due to other passengers

- Inconvenience: to what degree is it necessary to redirect from the original preferred route?

- Discomfort: invasions of personal space, loss of privacy.

- Distance: the experienced distance between the passenger's start and end point, weighted by the speed or desirability of individual movement segments.

The model calculates, based on these parameters, a cost for each passenger, and for each possible route. The model then chooses and adjusts the individual passenger (entity) route as required to minimize cost. The parameters for this objective function have been estimated from interviews with Metroselskabet and field observations by the authors. Note that the results assume a "greedy" passenger. No assumption is made that the passengers are taking COVID precautions. In that respect, the model results are "worst case".

The arrival rate of passengers to the station from outside (not arriving by train) is set in five minute increments. Bentley Legion implements a pseudo-Poisson arrival distribution. For each fixed modeling interval, where the desired number of arrivals is $n$, and the time duration of the interval is $e$, the software generates $n$ samples from the uniform distribution $(0, e)$. These variates are then sorted smallest to largest, and become the arrival times of the individual passengers from the start of the time interval.

The simulation allows continuous data collection. Experience in Copenhagen also is that nearly all passengers are able to board the first available train, so each train arrival-departure cycle represents a complete simulation iteration that is reasonably independent. In this analysis this is presumed to be true, and incidents of passengers left behind due to insufficient dwell time or crowding are not considered. A simulation run of 2.5 model hours allows for 87 departures in each direction with 104 second headway (half as many at Øresund). This provides a sample size of $n=87$ at Nørreport and Forum, and $n=43$ at Øresund.

As previously mentioned, three scenarios or traffic levels are simulated for each station. Each simulation approximates a peak travel cycle from 06:30 to 09:00. The scenarios are:

\section{- Pre COVID-19:}

Using passenger data from before the COVID-19 restrictions and impact of COVID-19. The passengers do not require any significant social distancing.

\section{- COVID-19 Base:}

Using passenger data from the COVID-19 period, where the total ridership is approximately $50 \%$ of the total ridership from before the COVID-19 outbreak. In this scenario, the passengers require an increased social distance. The social distancing is not enforced on trains, in train doors, and on escalators or staircases.

\section{- COVID-19 Projected:}

Using the same data and social distancing as in the COVID-19 Base scenario, but the passenger volume is increased by $50 \%$, so that the passenger traffic is equivalent to $75 \%$ of the total ridership from before the COVID-19 outbreak.

Bentley Legion offers a function called "direction modifiers", which allows the parameters of pedestrian behavior to be modified at selected locations and physical spaces within the model. This allows for relaxing of 
social distancing inside trains, at train doors, etc. Social distancing is enforced through Legion's “discomfort factor", which penalizes excessively close contact between pedestrians.

There are a few potential sources of error in the model. The first is the physical modeling of the station space. The station spaces have been modeled directly from pdf floor plans, which have been converted to a CAD file format, and there are small changes in the station dimensions from this conversion process. However, verification of a sample of measurements found very minimal deviation on the order of $0.005 \%$.

Another source of error is direction of flow. It is not really error, but a measure that is not recorded. The Danish health authorities differentiate between social distance between persons face to face, and social distance following in the same direction. This has led to the implementation of directional lanes of pedestrian traffic in shopping malls and other public spaces. The measures in this simulation are omnidirectional and not weighted for orientation of the passengers' bodies.

Finally, a significant source of error is presented in Figure 8 and is called "lockup". It is of course a wellknown phenomenon that under great stress, pedestrians can be forced into dangerous conditions where they compress themselves into infeasible spaces, leading to injury or even death. The most common examples are music concerts or sporting events.

Even though such conditions are not known at the traffic volumes simulated for the Copenhagen Metro, the implementation of social distancing in the model has the effect of creating such model results when they would not exist in reality. In such a condition, the simulation model ceases functioning because there is no algorithm to force pedestrians to reverse direction to clear the blockage. The incidence of lockup is random, and the solution in this study has been to simply run the model again. In most cases, the next random stream of events does not trigger lockup.

Figure 9 shows greater detail of this bottleneck at Nørreport. Approximately $80 \%$ of all Nørreport metro station traffic is destined to/from the S-bane transfer tunnel to the right in the figure. As a result, in the bottlenecks, marked in Figure 9, passengers are pushed in both directions, while the passengers waiting to board the train are pushed by the boarders and disembarkers. This often results in a complete lockup of the simulation. Direction modifiers are inserted at these bottlenecks to relax social distancing in the objective function, which greatly reduced the frequency of lockup.

\section{Results and findings}

The Transit Capacity and Quality of Service Manual (Transportation Research Board, 2013) proposes a pedestrian level of service (LOS) with six levels, A to F. These levels originate much earlier in the literature with Fruin (1987). Level A defines free flow, where pedestrians are largely unrestricted, and level F defines spaces that are tightly packed, with likely physical contact between pedestrians. In this paper, where the objective is social distancing, levels $\mathrm{E}$ and $\mathrm{F}$ are unacceptable and represent immediate failure. The color legend displayed in Bentley Legion displays these standard LOS levels.

The Transit Capacity and Quality of Service Manual (TCQSM) defines numerical LOS values for specific elements of infrastructure such as doors or walkways. TCQSM defines a level of service for walkways, and assigns numerical values of floor area per person to each level of service, ranging from 3.3 square meters at LOS A to 
less than a half square meter at LOS F. A social distancing of 1 meter would lie at LOS C. However, in this paper, there is a hard objective to measure, the specific measured separation between pedestrians, and this is not fixed in relation to available floor area. How the pedestrians flow and utilize the floor area can result in a much lower social distance than the available floor area might imply.

Five measures are used to analyze the adherence to social distancing: average density (passengers $/ \mathrm{m}^{2}$ ), maximum density (passengers $/ \mathrm{m}^{2}$ ), area utilization (time used), and 1- and 2-meter sphere violations (cumulative time that entities' spheres are violated by other entities). Figure 10 presents an example result for violations of the 2 meter sphere. The scale is in minutes accumulated over the model run. For example, the blue zone represents a maximum of five minutes of violated contact accumulated through the 2.5 hour run. Note that these are momentary violations in most cases, as passengers are stationary only when waiting to board trains.

Somewhat as expected, the stations are most affected according to their traffic volume. Øresund station is the least affected by the social distancing goals. Passenger density is generally low, and violations of the social distancing spheres are rare. The recommendation for Øresund station would be to take no action.

Forum station, the intermediate traffic density example, experiences stress at the escalators, particularly sphere violations. Area utilization is low. These results were consistent across the three scenarios. No actions are recommended for Forum station.

Nørreport station, the high traffic example, exhibits significant sphere violations, as seen in Figure 10. The design of the station should be quickly explained. From the left, there is an elevator at the extreme end of the platform, to surface level. Then, at approximately the third platform door there is a set of escalators to surface station level. At the sixth (last) platform door, there is another set of escalators to surface station level. To the far right, is a set of escalators and a ramp to the S-bane (suburban railway) platforms, called the "transfer tunnel". Under and behind these escalators is an elevator also leading to the S-bane. Note that if social distancing is relaxed to a one meter separation, the distancing violations nearly disappear at COVID traffic volumes, as shown in Figure 11 , but social distancing will be a concern if traffic returns to pre-COVID levels.

For all scenarios, there is a bottleneck between the escalator set at door six and the platform doors (also shown in Figure 9), as well as at the exit from the station to the transfer tunnel. These bottlenecks can be clearly seen at the 1- and 2-meter sphere breaches and especially at 2-meter sphere in the scenario during covid-19 these are evident. Face to face interactions are a critical problem because passengers are moving in both directions, boarding and alighting from trains and transferring to and from the S-bane. Only on the escalators is one assured of face to back positioning. Similarly, the area around the exit to the transfer tunnel is a problem area as travelers here may encounter face-to-side interactions that are still worse than face-to-back interactions but better than face-to-face interactions.

For comparison, results for average density at Nørreport station are shown in Figure 12. Note that the distribution of social distancing violations and the areal density are not the same. A social distancing of 2 meters is approximately an area of 0.178 persons per square meter (https://en.wikipedia.org/wiki/Circle_packing), corresponding to the blue legend of Figure 12. Under COVID conditions, most of Nørreport would appear to satisfy LOS C, "walking speeds freely selected", but clearly from Figure 10 the social distancing goals are not attained.

A number of recommendations can be offered for Nørreport station, of varying degrees of difficulty. The first would be greater enforcement of directionality in passenger flow. This is a challenge because of the necessity for 
passenger flow to/from the transfer tunnel to walk directly past waiting passengers at door six. The second would be increasing the capacity of the escalators to the transfer tunnel. The transfer tunnel escalators are under-sized for the demand. There are two escalators in both directions to the surface, but only one escalator to the transfer tunnel. An extreme and expensive solution would be to remove entirely the right set of surface escalators, to provide more lanes for pedestrian traffic flow on the platform. This is unlikely because the original framework for this study is to evaluate minimal infrastructure changes.

One of the assumptions in the model and the choice of a single simulation run with 87 departures is that no passengers are left behind after train departure, and that thus the 87 departures are reasonably independent iterations of the simulation. The simulation is able to track the count of passengers onboard each train departure. From this data, it is possible to indicate exactly which departures could not meet demand, both before arrival at the station and after departure.

The capacity limit used for the capacity assessments is a maximum of 279 passengers/train Pre-COVID-19, , and 160 passengers/train for the scenarios COVID-19 Base and COVID-19 Projected. After analysis of the data logs from the runs, only two single departures exceeded train capacity. That is to say, three scenarios times (two stations times 87 departures times two directions plus one station with 43 departures times two directions), equal to 1302 departures, contain only two departures where the train capacity is exceeded and passengers are left behind.

\section{Conclusion}

Public transport is a critical service in Copenhagen, because many residents do not own a car, and in any event car travel is not practical in the city center due to narrow roads and lack of parking. The COVID-19 pandemic presents a challenge and hard constraint, because public health concerns demand a reduced density of persons both at stations and on transport vehicles. Danish public health authorities have established a minimum 1 meter social distancing policy in public spaces with a further preferred 2 meter social distance. This study simulates passenger pedestrian flow in three representative stations of the Copenhagen metro to determine if these goals can be attained, and if any physical changes should be made.

The study is conducted with a micro-simulation in Bentley Legion of the passenger flow in three representative stations, with small, medium, and large traffic flows. The model simulates peak morning traffic flow over a 2.5 hour interval with pedestrian parameters representing commuters. Each simulation run represents contains a minimum of 86 train departures, and the complete result set contains 1302 departures. The simulation is agentbased, and the individual objective function is minimum cost according to walking distance, comfort, and frustration.

Some of the challenges in simulating this pedestrian flow included modeling direction of flow and lockup. Bentley Legion does not distinguish between social distance violations face-to-face and social distance violations face-to-back. Danish public health authorities have advised that face-to-back orientation is safer and preferable when there is high density foot traffic, and has implemented directional paths in public spaces and walkways to encourage this. The data from the simulation model does not distinguish between violations of social distance face-to-face and face-to-back.

Lockup is a condition where a simulation model moves entities into positions where no further forward movement of the simulation is possible. The stations contain areas of opposing pedestrian flow, and when the 
social distancing parameters are enforced, the movement of the required passengers becomes infeasible and the simulation run locks up. It is necessary to relax the social distance parameters in these selected bottleneck zones.

The results show that for the majority of stations, the physical infrastructure and the expected traffic flow are compatible with the social distancing goals. However, for a few of the highest demand stations, particularly those that serve as intermodal hubs, there are great difficulties in achieving the desired social distancing measures. In particular, the intermodal hub station of Nørreport does not possess corridors and escalators which are distributed correctly according to the pedestrian flow. This station is underground and it is unfortunately not easy to change this infrastructure.

The COVID-19 pandemic is a fast changing problem and it is very difficult to predict future needs or status. Predictions made six months ago have in many cases not become reality. Further research on this problem could address the conversion of existing fixed stairways to escalators. This is an infrastructure investment, but more reasonable because it does not require an increase in the physical dimensions of the station or changes to the patterns of flow. Another possible solution to evaluate would be synchronizing the opposing trains on the same platform, so that they arrive and depart on as near opposite time cycles as possible. Of course, this solution is only valid for a single station on a railway line, as then the departure times at the other stations are fixed in synchronization with the chosen station.

"Thinking outside the box", another completely different solution would be to reroute passengers away from Nørreport station. Denmark operates a public transport planning application, Rejseplanen, which is widely adopted. It could be possible to reprogram the routing algorithm to avoid Nørreport during peak travel times.

Acknowledgements: the authors wish to thank Metroselskabet I/S for their support and cooperation in performing this research

Data availability statement: Some or all data, models, or code that support the findings of this study are available from the corresponding author upon reasonable request.

- Passenger count statistics

- Drawings of the stations

\section{References}

Alexandersson, Stina \& Emmi Johansson (2013) Pedestrians in microscopic traffic simulation. MSc thesis, Chalmers University of Technology, Gothenburg.

Azadpeyma, A., \& Kashi, E. (2019). Level of Service Analysis for Metro Station with Transit Cooperative Research Program (TCRP) Manual: A Case Study—Shohada Station in Iran. Urban Rail Transit, 5(1), 39-47. https://doi.org/10.1007/s40864-018-0098-0

Bentley Systems. (2019). "Legion Simulator Help." Retrieved 3 March 2021 from: https://docs.bentley.com/LiveContent/web/LEGION\%20Simulator\%20Help-v2/en/GUID-E6FFDCA7-39CF47F8-A0F1-7DF015290C7A.html

Dubroca-Voisin, M., Kabalan, B., \& Leurent, F. (2019). On pedestrian traffic management in railway stations: 
Duives, D. C., Daamen, W., \& Hoogendoorn, S. P. (2013). State-of-the-art crowd motion simulation models. Transportation Research Part C: Emerging Technologies, 37, 193-209. https://doi.org/10.1016/j.trc.2013.02.005, 10.1016/j.trc.2013.02.005

Finnis, K. K., and D. Walton. (2008). "Field Observations to Determine the Influence of Population Size, Location and Individual Factors on Pedestrian Walking Speeds." Ergonomics 51 (6): 827-42. https://doi.org/10.1080/00140130701812147.

Fruin, John J. (1987) Pedestrian Planning and Design. Elevator World, ISBN 9781886536012.

Grontmij Carl Bro (2009) Grontmij Carl Bro and Legion real-time 3D simulation model of the Station. Available from: http://bentleyuser.dk/sites/default/files/b6_-_crowd__pedestrian_simulation_norreport_station.pdf [accessed 26 August, 2021]

Herrstedt, L. (2012). ’Nordic Human Factors Guideline Ganghastigheder.” Trafitec Aps, Søborg, Denmark Jacobs, H. (1967). “To count a crowd.” Columbia Journalism Review 6, 36-40.

Little, John D. C. 1961. “A Proof for the Queuing Formula: L= $\lambda$ W.” Operations Research 9 (3): $383-87$. https://doi.org/10.1287/opre.9.3.383.

Metroselskabet I/S. (2018). "Metroen i tal". Retrieved 11 November 2020 from: https://m.dk/ommetroen/metroen-i-tal/passagertal/

Pedersen, C. Kirchoff., and Center for Trafik og Transport. (2003). ’Holdetid På Togstationer.” CTT/ Atkins

Seriani, S., \& Fernandez, R. (2015). Pedestrian traffic management of boarding and alighting in metro stations. Transportation Research Part C: Emerging Technologies, 53, 76-92. https://doi.org/10.1016/j.trc.2015.02.003

Sun, L. S., Cui, L., Rong, J., Zhao, P. F., \& Luo, W. (2016). Subway Corridor Curve Effects on Pedestrian Characteristics Based on the Legion Studio-Enabled Simulation. Cictp 2016 - Green and Multimodal Transportation and Logistics - Proceedings of the 16th Cota International Conference of Transportation Professionals, 965-975. https://doi.org/10.1061/9780784479896.088

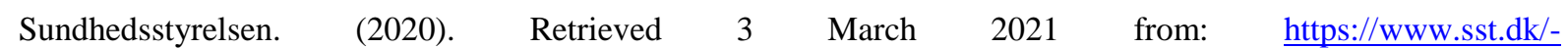
/media/Udgivelser/2020/Corona/Forebyggelse-af-smittespredning/Forebyggelse-af-smittespredningpublikation.ashx

Transportation Research Board (eds.) (2013) Transit capacity and quality of service manual, 3rd edn. The National Academies Press, Washington. https://doi.org/10.17226/24766 


\begin{tabular}{|l|c|c|c|c|}
\hline Train direction & \multicolumn{2}{|c|}{ Towards Airport } & \multicolumn{2}{c|}{ Towards Vanløse } \\
\hline Station & \multicolumn{2}{|c|}{ Nørreport } & \multicolumn{2}{c|}{ Nørreport } \\
\hline Passenger direction & In & Out & In & Out \\
\hline door 1 & $27 \%$ & $33 \%$ & $18 \%$ & $13 \%$ \\
\hline door 2 & $16 \%$ & $19 \%$ & $12 \%$ & $11 \%$ \\
\hline door 3 & $17 \%$ & $15 \%$ & $6 \%$ & $13 \%$ \\
\hline door 4 & $14 \%$ & $12 \%$ & $15 \%$ & $17 \%$ \\
\hline door 5 & $12 \%$ & $8 \%$ & $24 \%$ & $15 \%$ \\
\hline door 6 & $14 \%$ & $13 \%$ & $25 \%$ & $30 \%$ \\
\hline Total & $100 \%$ & $100 \%$ & $100 \%$ & $100 \%$ \\
\hline
\end{tabular}

465

\begin{tabular}{|l|c|c|c|c|}
\hline Train direction & \multicolumn{2}{|c|}{ Towards Airport } & \multicolumn{2}{c|}{ Towards Vanløse } \\
\hline Station & \multicolumn{2}{|c|}{ Forum } & \multicolumn{2}{c|}{ Forum } \\
\hline Passenger direction & In & Out & In & Out \\
\hline door 1 & $19 \%$ & $8 \%$ & $16 \%$ & $27 \%$ \\
\hline door 2 & $9 \%$ & $10 \%$ & $16 \%$ & $17 \%$ \\
\hline door 3 & $4 \%$ & $7 \%$ & $18 \%$ & $20 \%$ \\
\hline door 4 & $20 \%$ & $15 \%$ & $19 \%$ & $9 \%$ \\
\hline door 5 & $24 \%$ & $19 \%$ & $13 \%$ & $20 \%$ \\
\hline door 6 & $25 \%$ & $40 \%$ & $18 \%$ & $8 \%$ \\
\hline Total & $100 \%$ & $100 \%$ & $100 \%$ & $100 \%$ \\
\hline
\end{tabular}

466

\begin{tabular}{|l|c|c|c|c|}
\hline Train direction & \multicolumn{2}{|c|}{ Towards Airport } & \multicolumn{2}{c|}{ Towards Vanløse } \\
\hline Station & \multicolumn{2}{|c|}{$\varnothing$ resund } & \multicolumn{2}{c|}{$\emptyset$ resund } \\
\hline Passenger direction & In & Out & In & Out \\
\hline door 1 & $27 \%$ & $33 \%$ & $18 \%$ & $13 \%$ \\
\hline door 2 & $16 \%$ & $19 \%$ & $12 \%$ & $11 \%$ \\
\hline door 3 & $17 \%$ & $15 \%$ & $6 \%$ & $13 \%$ \\
\hline door 4 & $14 \%$ & $12 \%$ & $15 \%$ & $17 \%$ \\
\hline door 5 & $12 \%$ & $8 \%$ & $24 \%$ & $15 \%$ \\
\hline door 6 & $14 \%$ & $13 \%$ & $25 \%$ & $30 \%$ \\
\hline Total & $100 \%$ & $100 \%$ & $100 \%$ & $100 \%$ \\
\hline
\end{tabular}

467

468 Table 1: Distribution of preferred door at Nørreport, Forum and Øresund stations, "in" and "out" refers to entering 469 or exiting the train. 


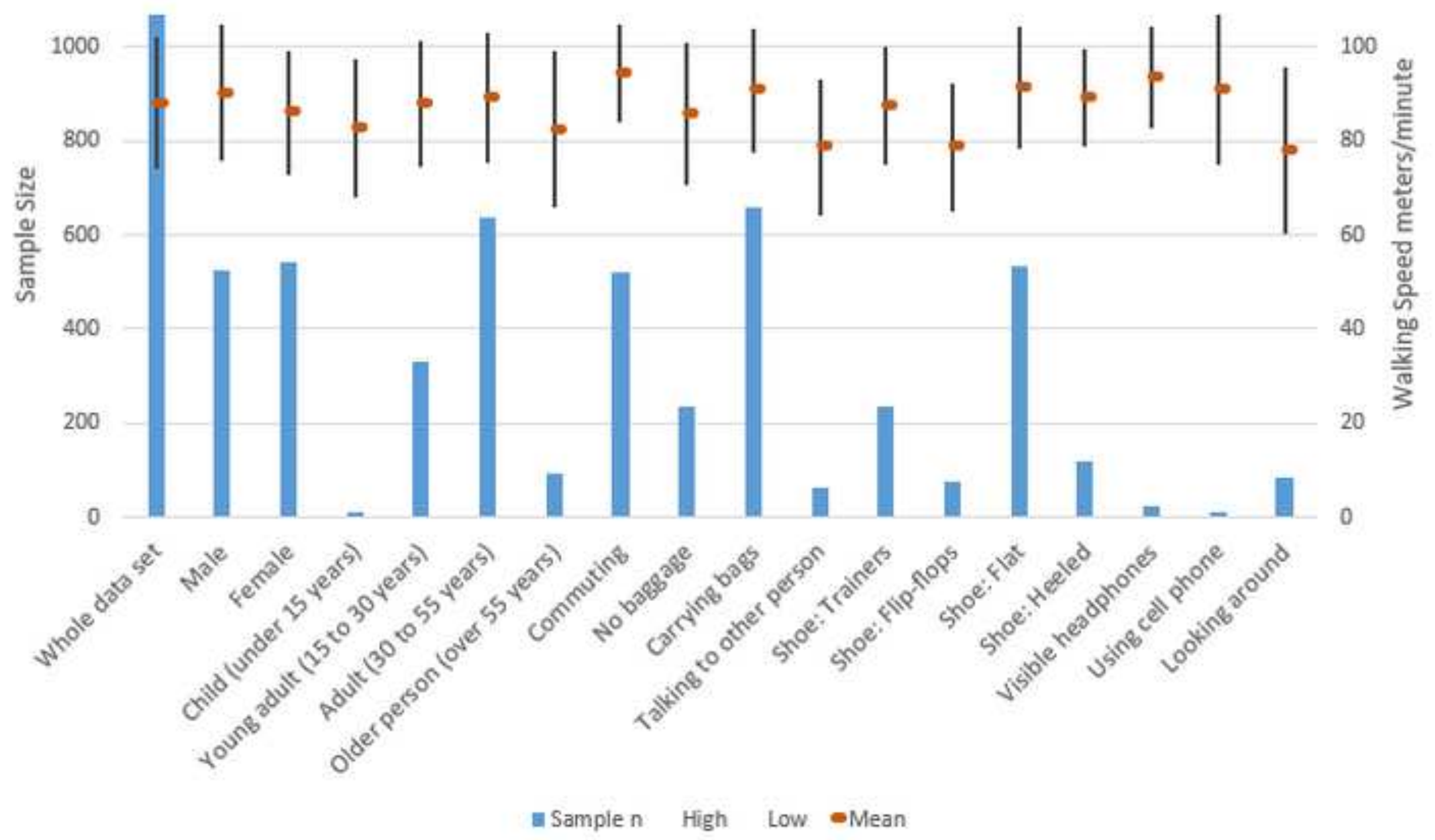




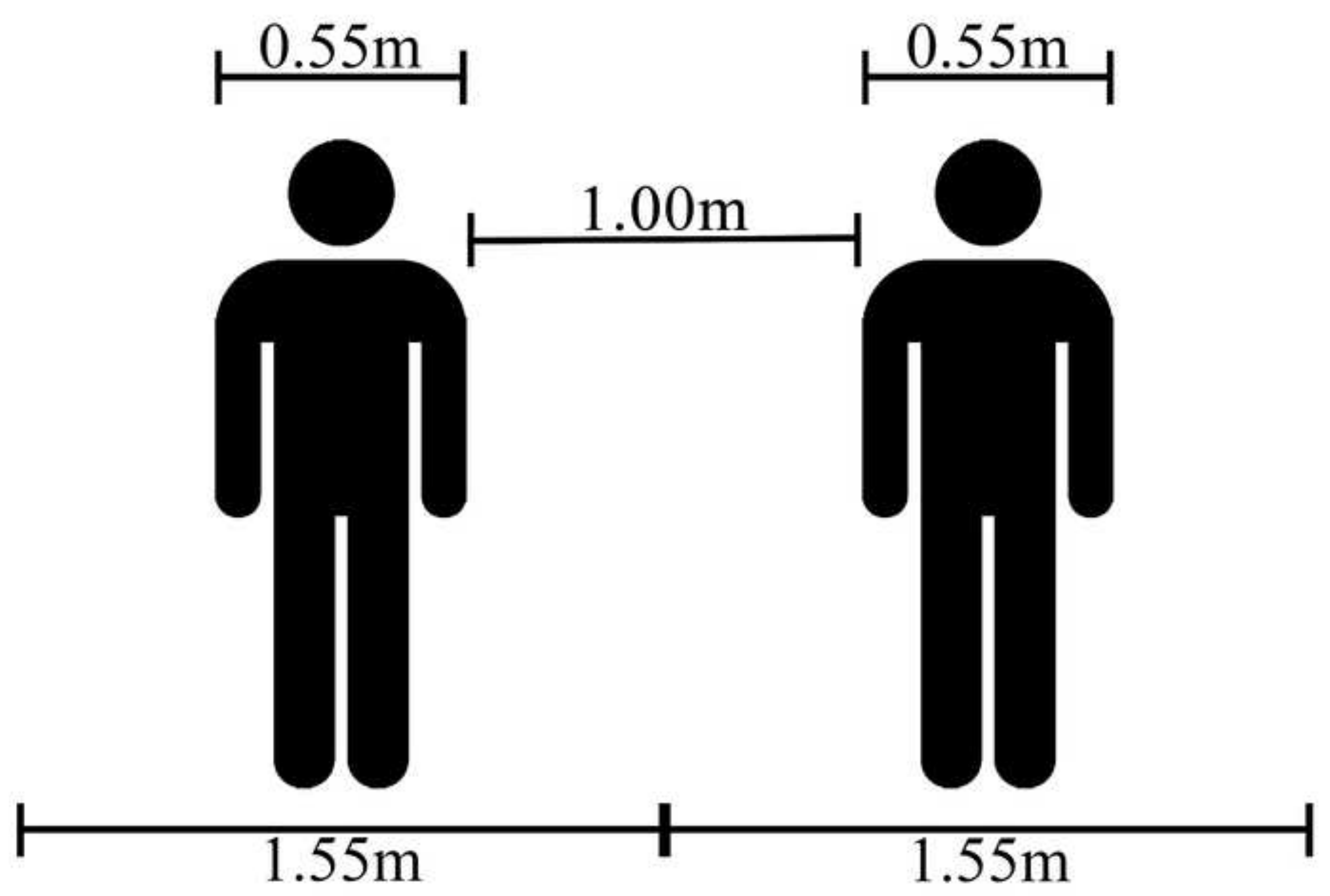




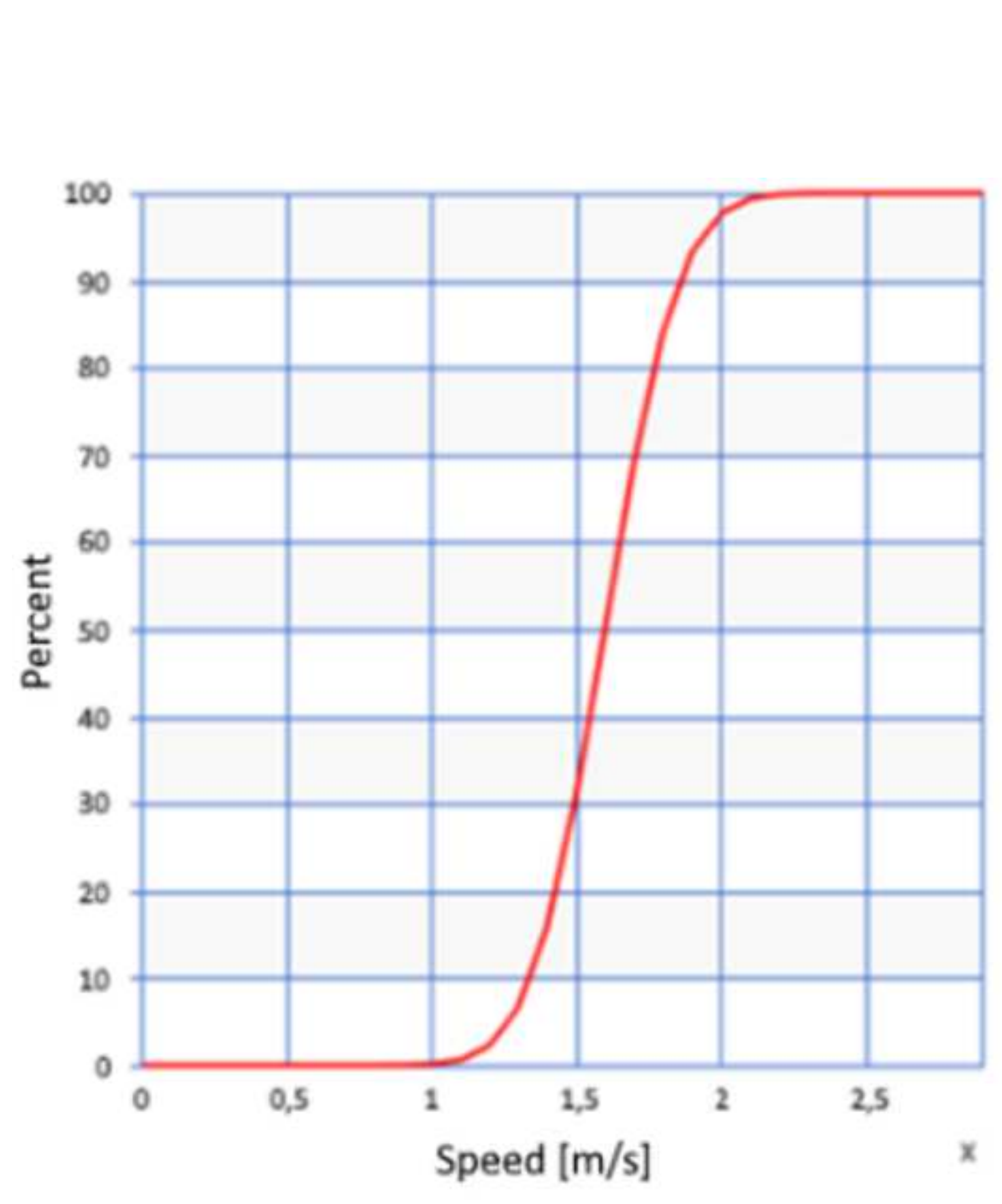

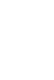




\section{Average weekday passenger number 2018}

\subsection{0}

50.000

40.000

30.000

20.000

10.000

0

四
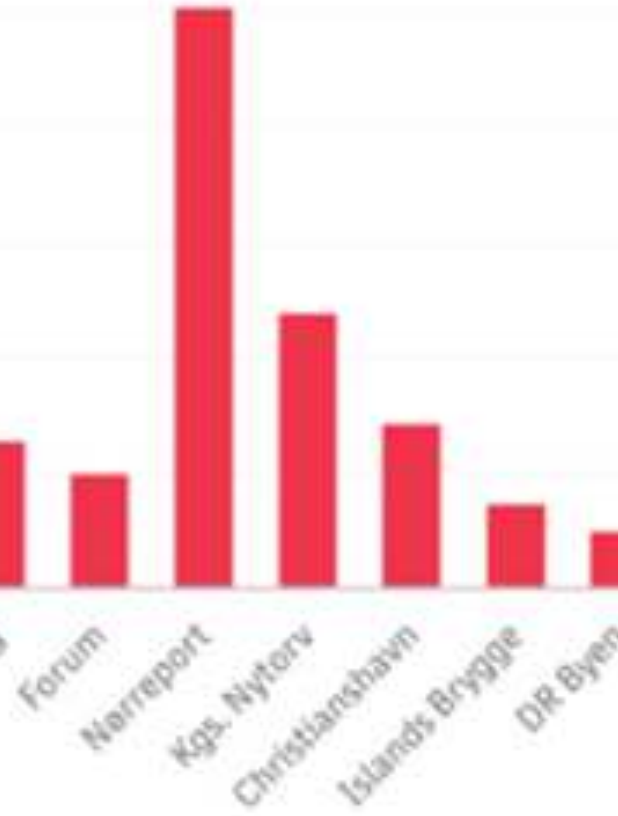

$a^{8} s^{4}$

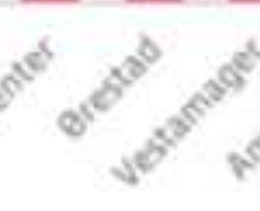




\section{Passenger development in the M1/M2, during COVID-19}

300.000

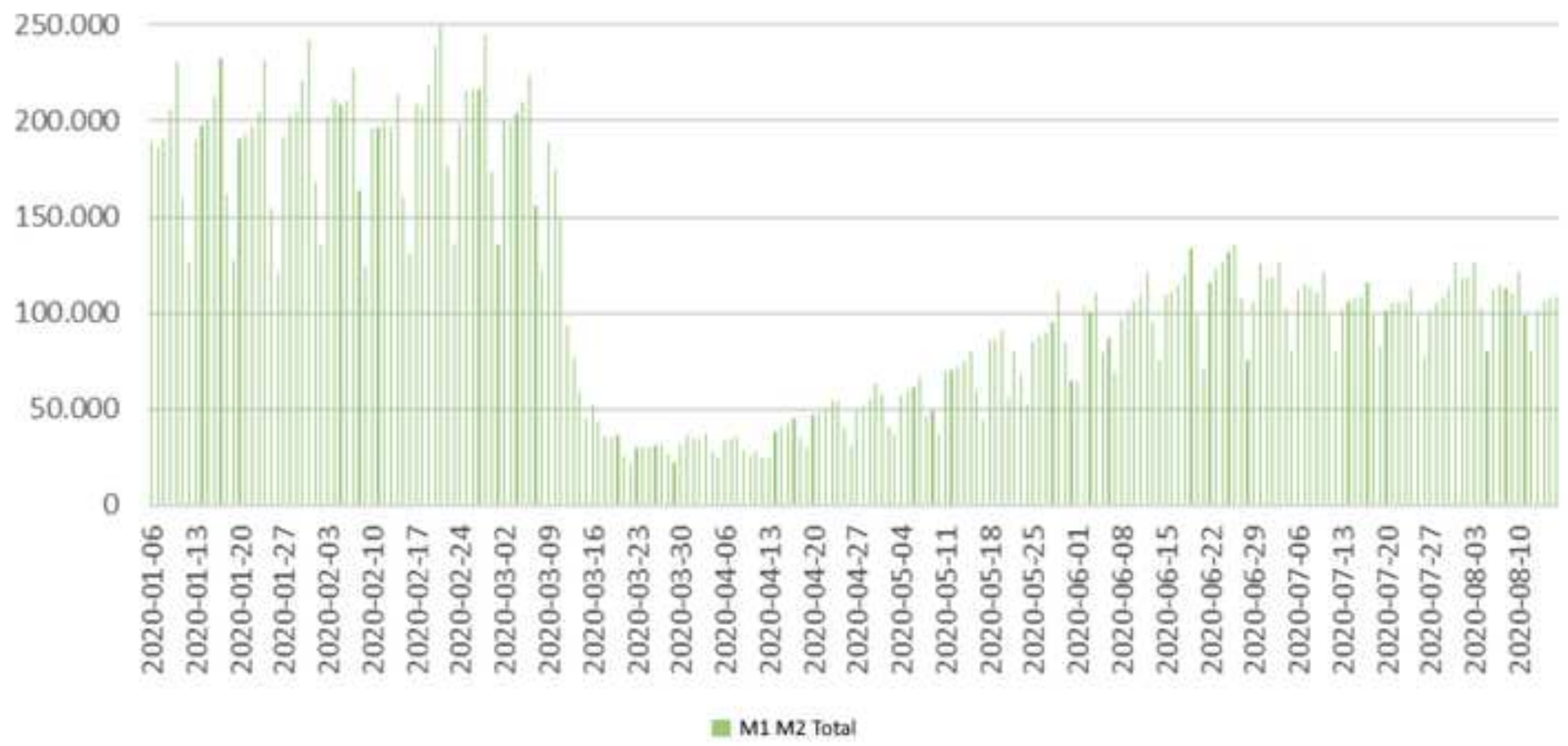




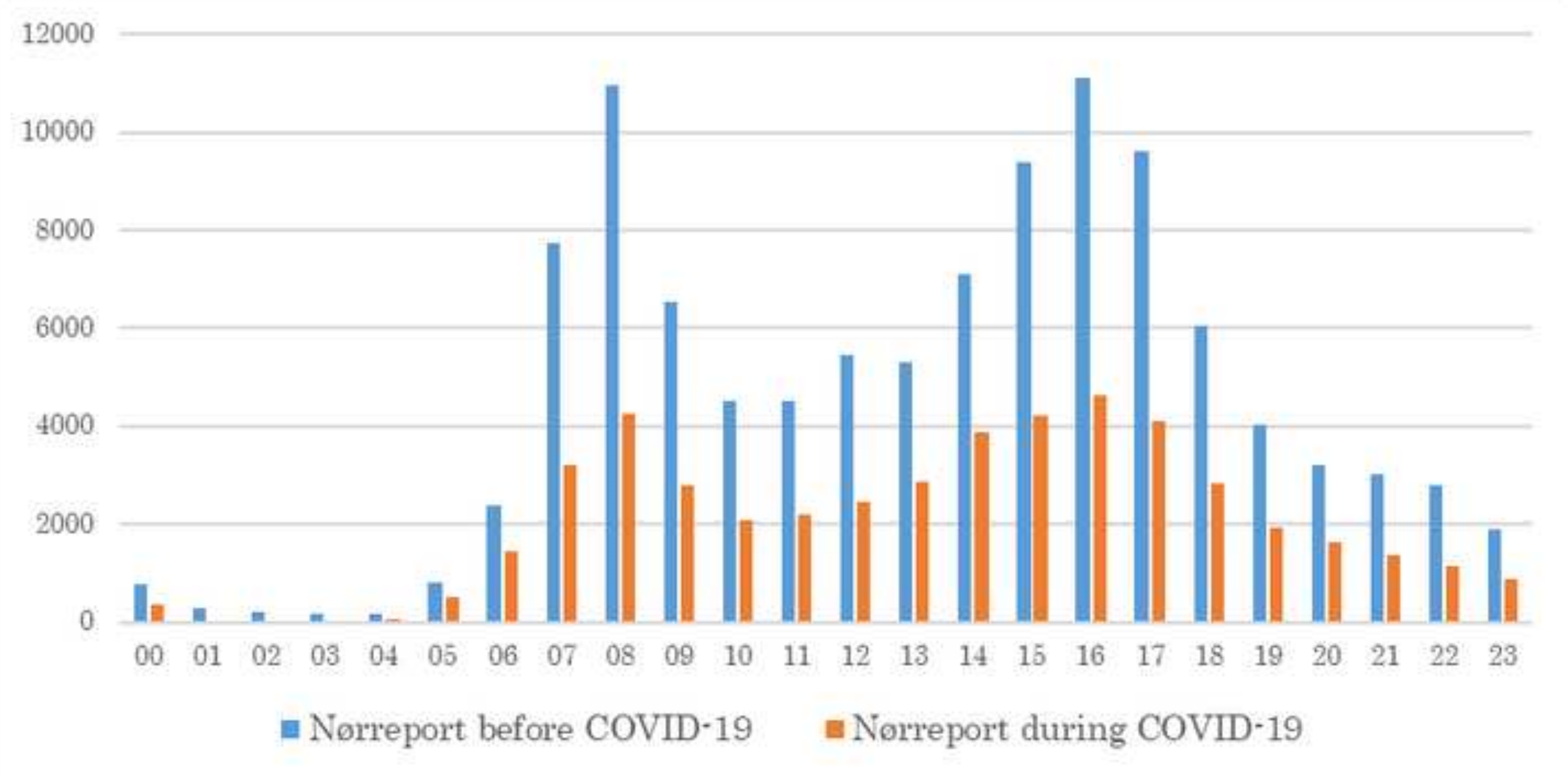




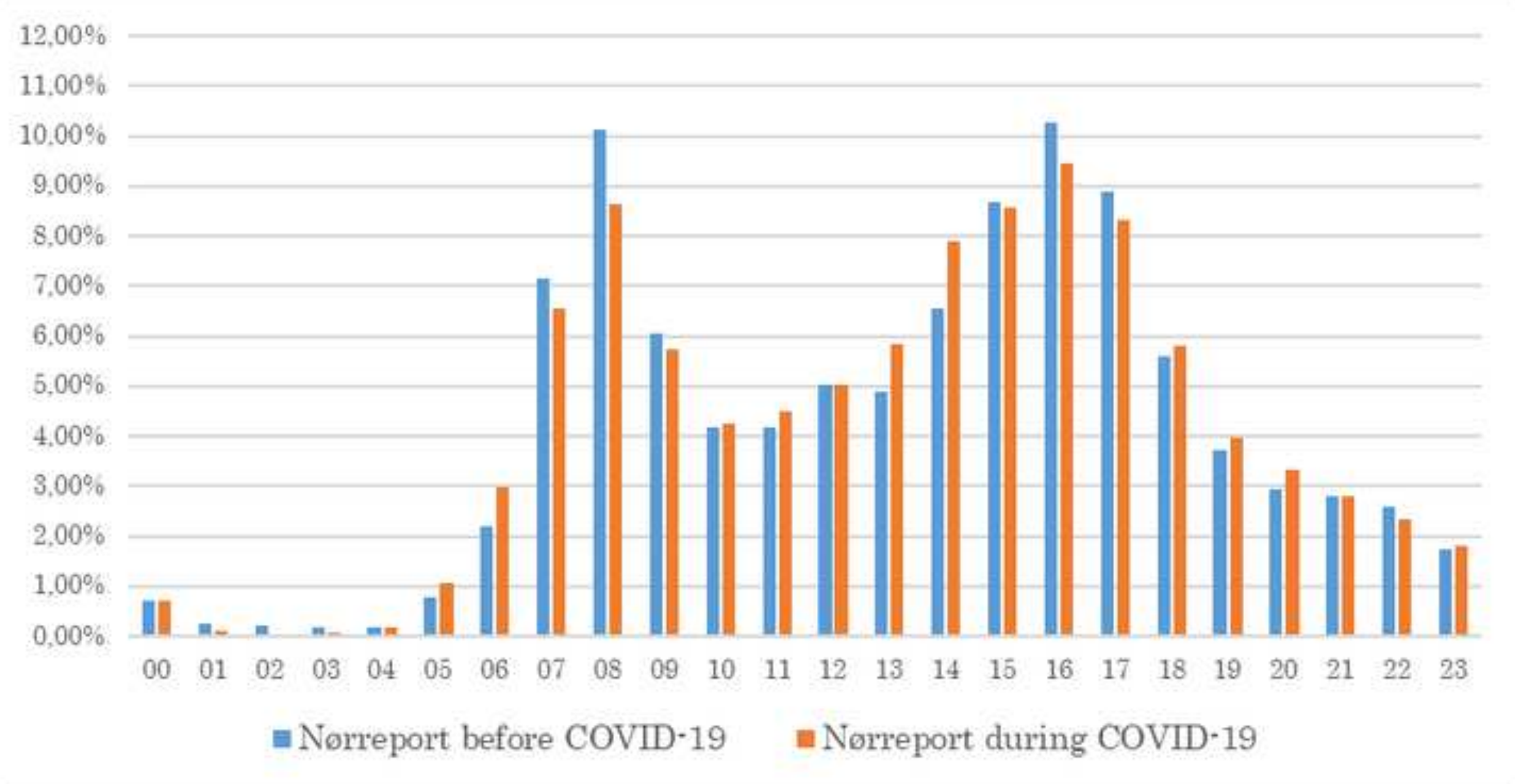




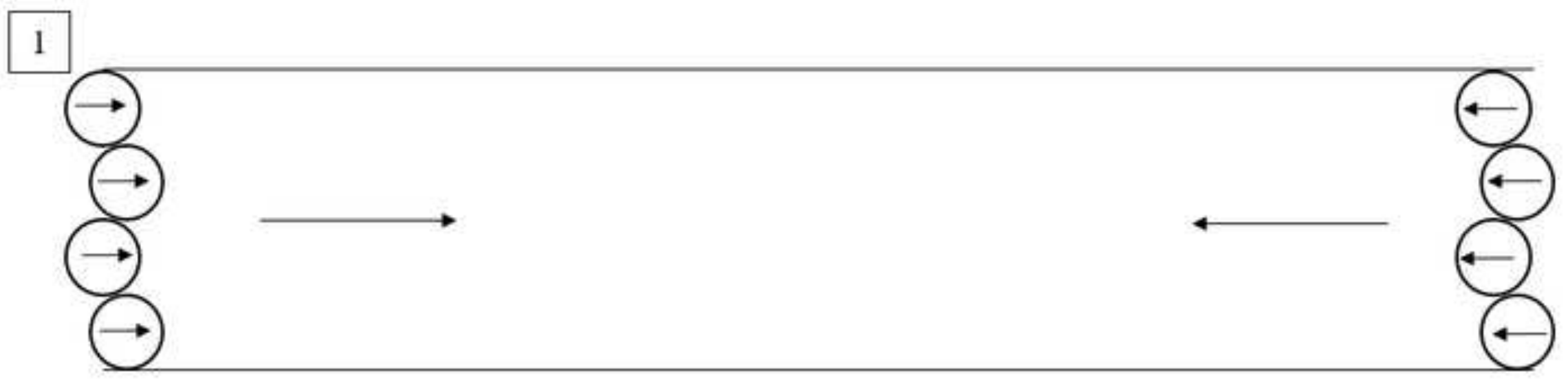

2

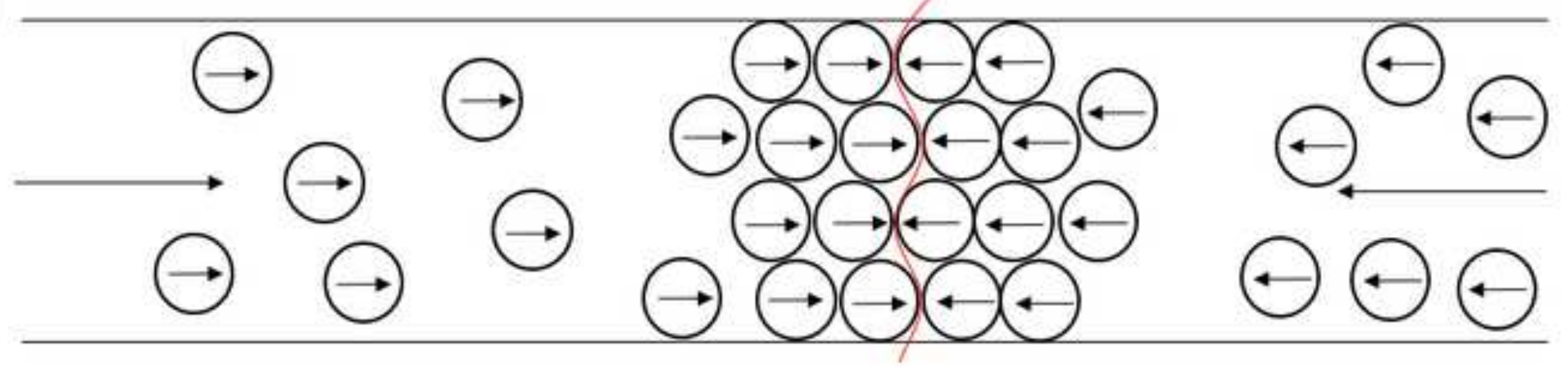




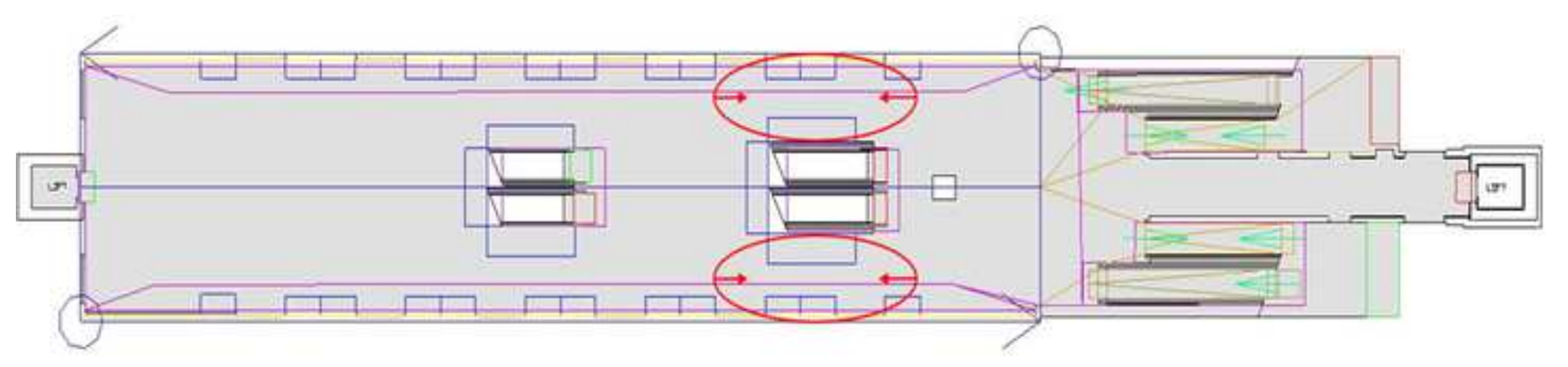




\section{Nørreport}

$2 \mathrm{~m}$ sfære

Før

covid-19

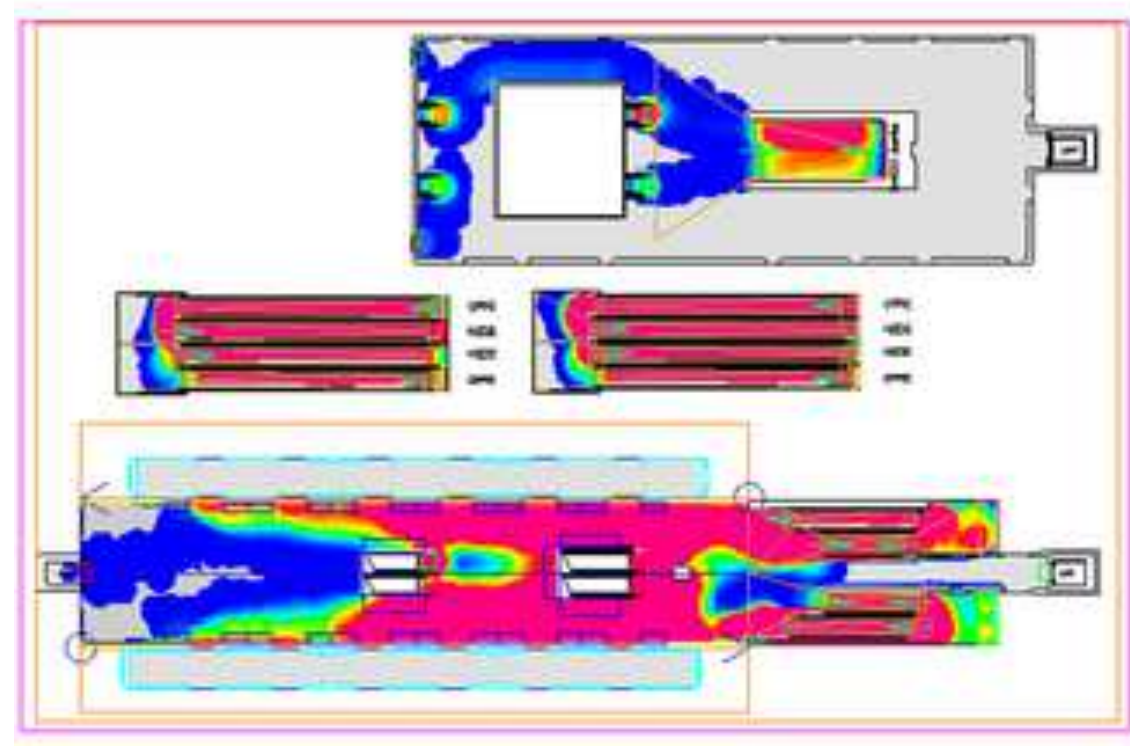

Under

covid-19

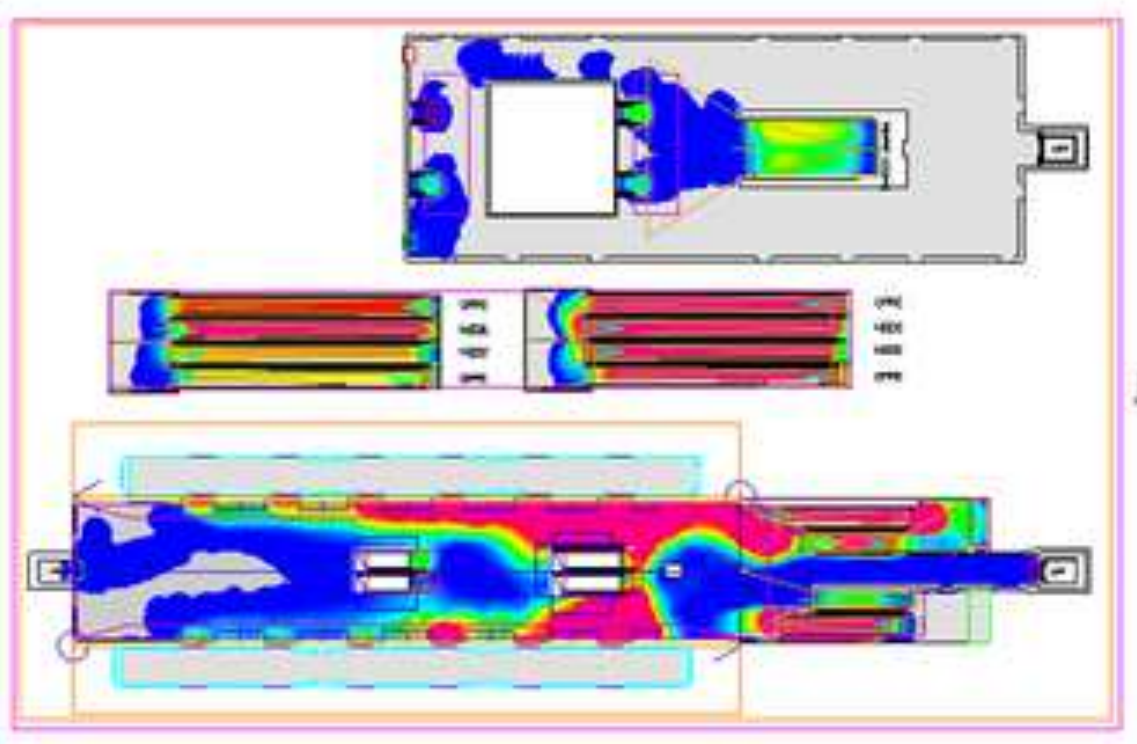

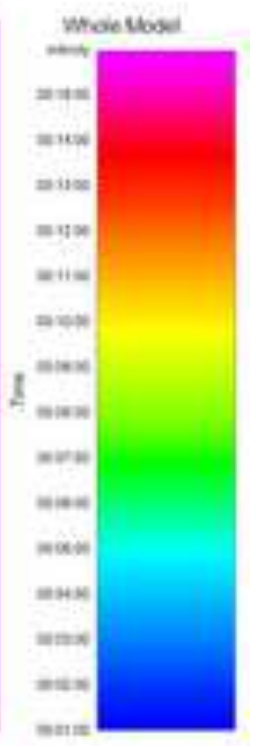


Nørreport

1m sfære

Før

covid-19

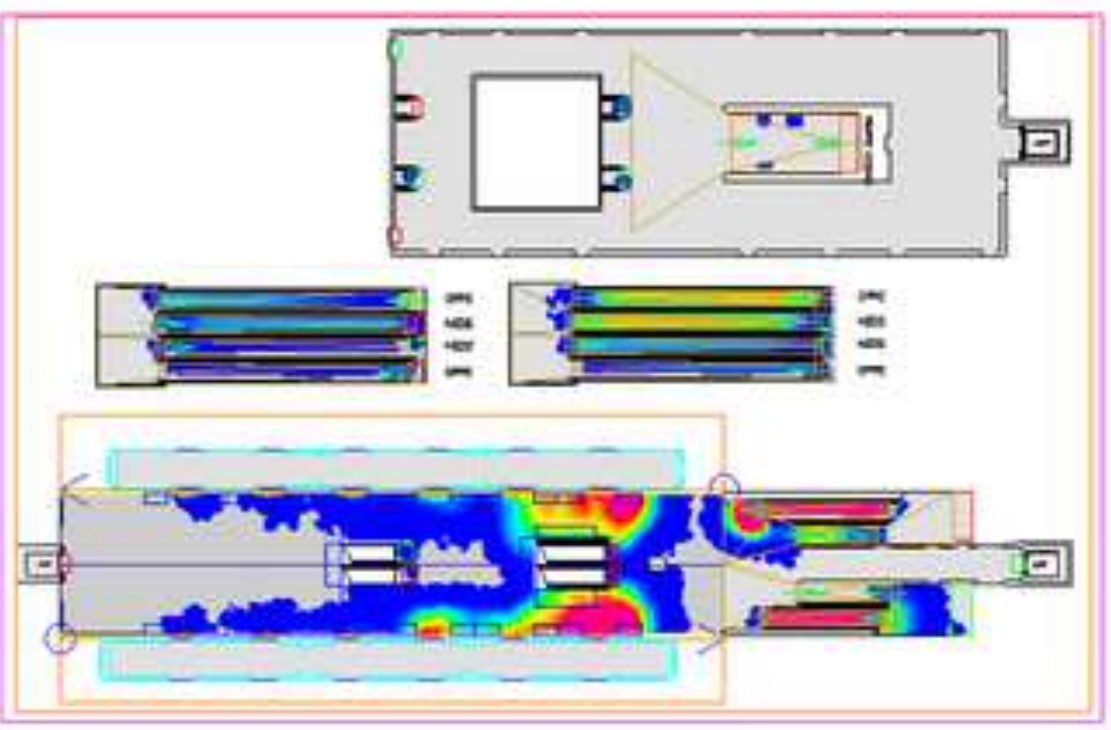

Under

covid-19
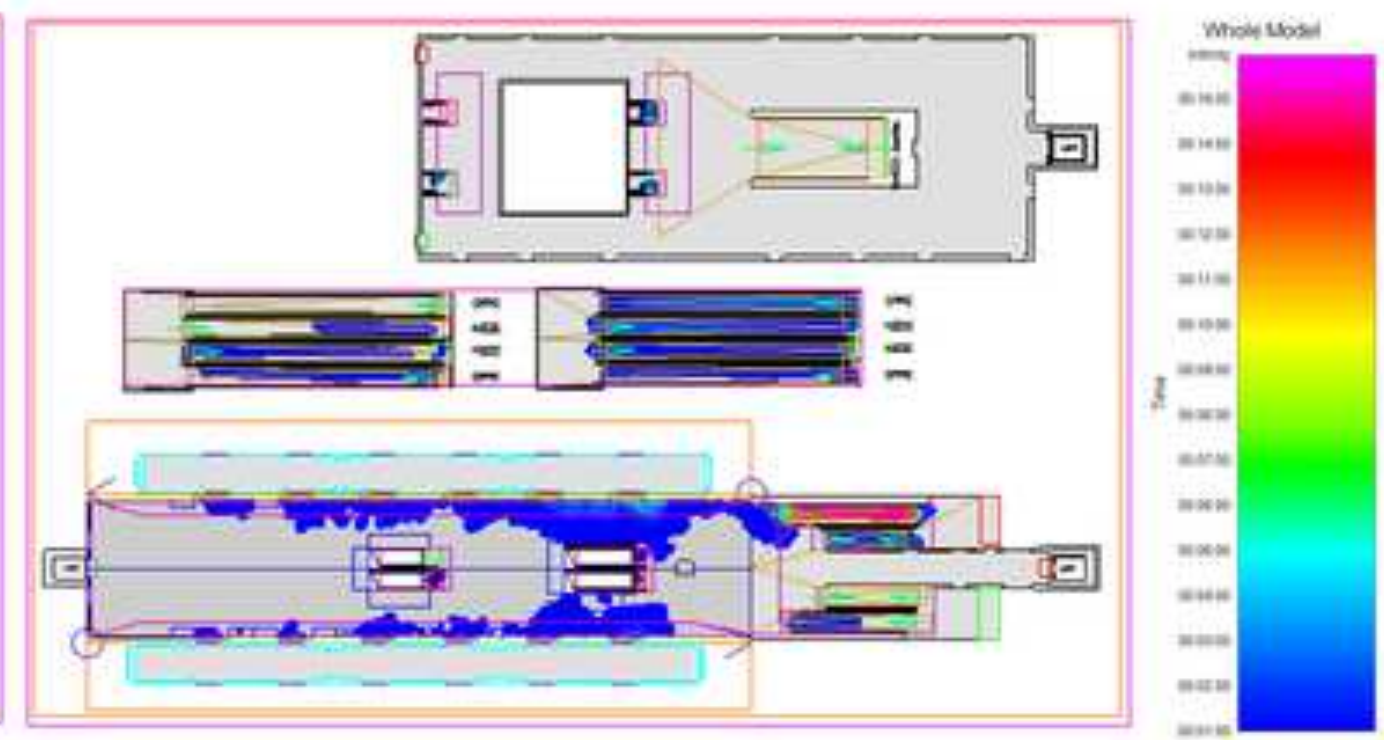


\section{Nørreport}

Gennemsnitlig densitet

\section{Før \\ covid-19}

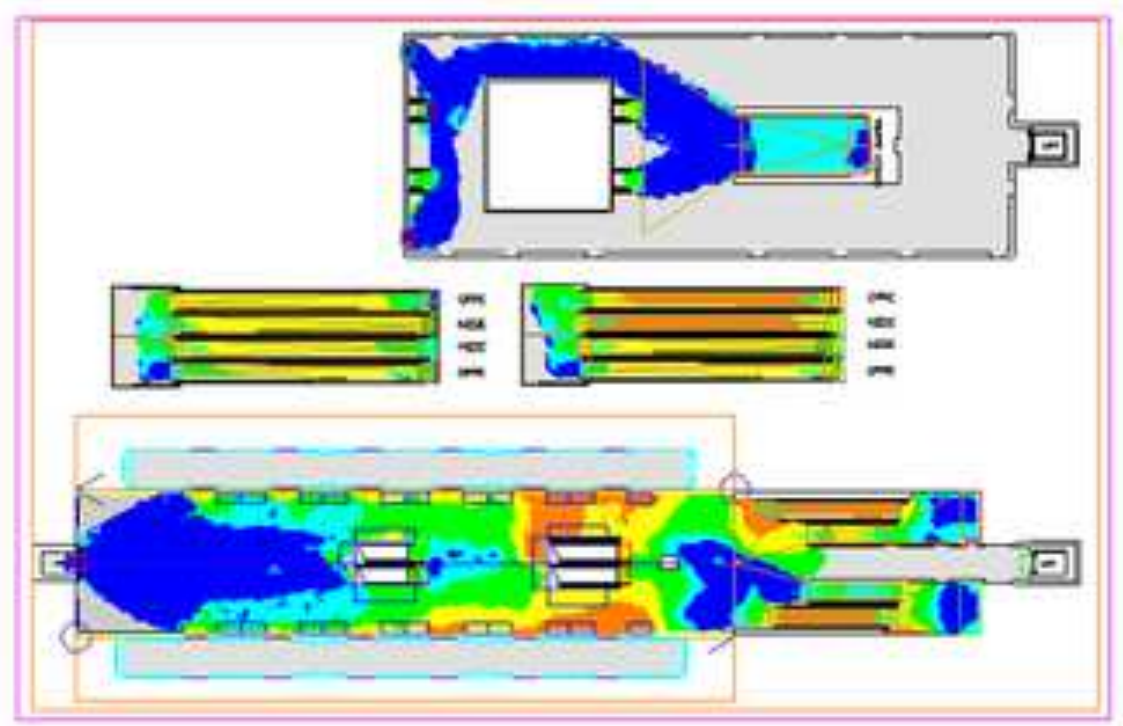

Under

covid-19
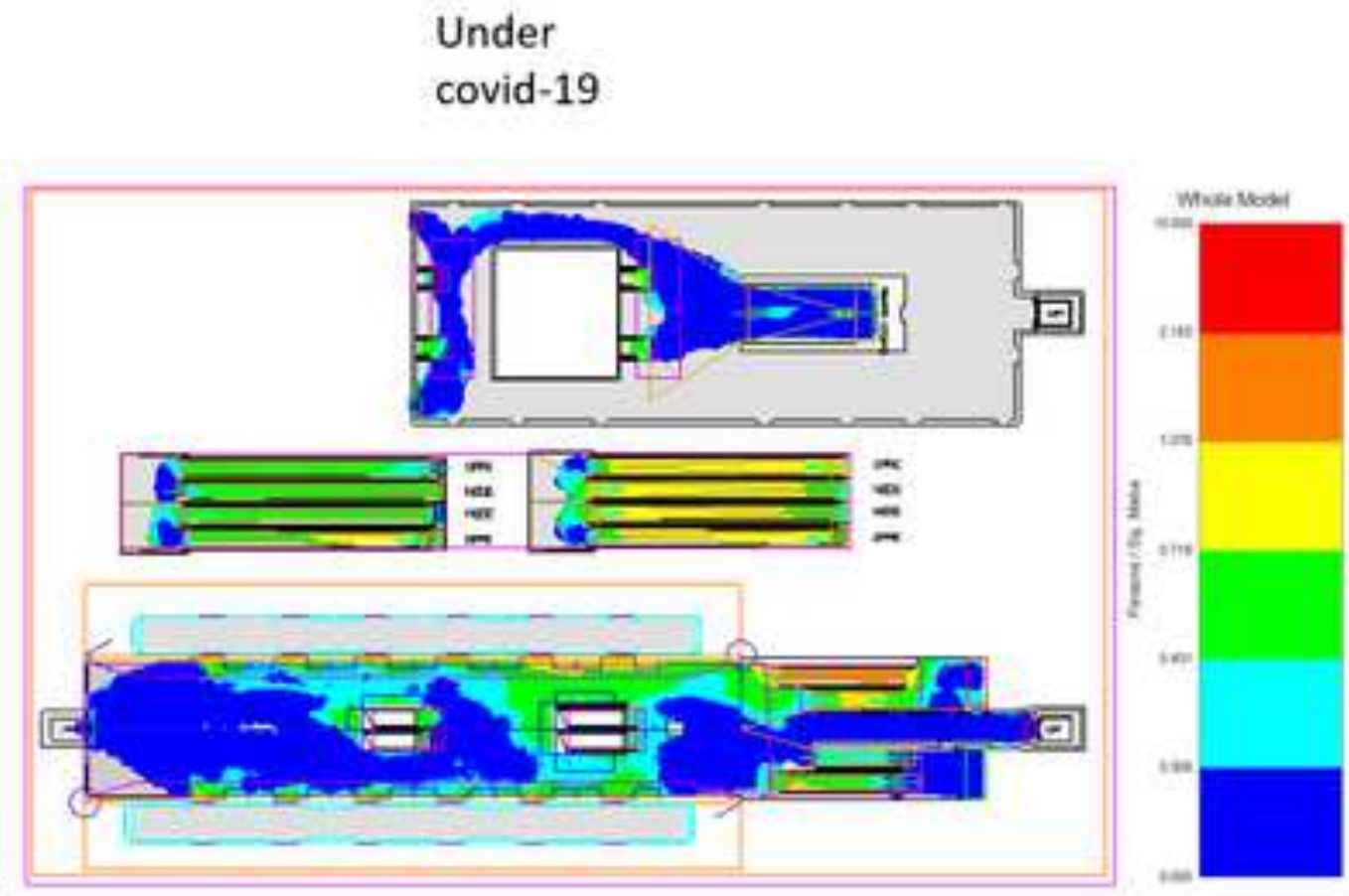


\section{Figure Captions}

2 Figure 1: Walking speed observations (Finnis and Walton, 2008). "High" and "low" ranges are one standard

3 deviation from plotted mean. Column represents sample size.

4

5 Figure 2: Cumulative distribution of passenger walking speed as applied in simulation model

6

7 Figure 3: Desired passenger density when enforcing COVID-19 precautions.

Figure 4: Average weekday passenger count by station, M1/M2 lines, 2018. (Metroselskabet, 2018)

Figure 5: Total passengers on M1/M2 during the initial COVID-19 period.

Figure 6: Hourly passenger counts before and after COVID-19 at Nørreport.

Figure 7: Hourly passenger counts, before and after COVID-19, normalized by aggregate totals, Nørreport.

Figure 8: Demonstration of the principle of "Lockup". Part 1: pedestrians approaching each other at end of a

bottleneck. Part 2: equally size groups of passengers fill the bottleneck in opposing directions, blocking all flow.

Figure 9: Location of particularly difficult bottlenecks and passenger directions at Nørreport station.

Figure 10: Violations of the 2-meters sphere in Nørreport station

Figure 11: Violations of the 1-meters sphere in Nørreport station 